\title{
Analytical and Numerical Simulation Well Test Models
}

\author{
PACH, Ferenc, MOL PIc. Hungary \\ GILICZ, András, MOL Plc. Hungary
}

Paper presented at the 5th European Conference on the Mathematics of Oil Recovery, Leoben, Austria, 3-6

Sept. 1996

\section{ABSTRACT}

A new analytical i.e. - for more complex cases - a numerical model is proposed for evaluating well tests. The analytical solution can be applied to special cases, whereas the numerical model is fully explicit, and can be used to complex well patterns, arbitrary grid geometry with general shape signal generation.

\section{INTRODUCTION}

The knowledge of petrophysical reservoir parameters is important, as they strongly influence production planning and analysis. Conventional coring is expensive, further the extrapolation of the point like sampling of the reservoir is risky. Well test are used to complete or check core analysis results.

Literature provides several evaluation methods, which reliability depends on the applied mathematical and physical conditions. The known methods are based practically on the exponential integral (Ei) solution, which however is only a special case.

We consider in our model the reservoir-aquifer system thermodynamically as an elastic, deformable system, which deforms under certain impacts i.e. influences. Prior to exploration the system is in rest and in equilibrium. Under the impact of production - which is a series of influences - the system is diverted from its equilibrium state. Systems diverted from their equilibrium state and driven by natural laws move always voluntarily, deterministically, and irreversible towards their current equilibrium state, which can possibly change with time.

The response given by the system to impacts is determined by physical laws, like conservation of mass, energy and impulse, the change of impulse and the continuity law. The validity of natural laws is independent from the size of the system, from its geometrical shape, fluid composition, etc.

The shape of the reservoir-aquifer systems is versatile and generally irregular. Despite of this, investigated systems are supposed to be radial/cylindrical in order that analytical solutions can be applied. This is why natural laws governing the systems and expressed as partial differential equations (PDE) are investigated in radial/cylindrical coordinates.

The pressure distribution of slightly compressible systems can be determined by means of the continuity law:

$$
\operatorname{div}\left(\frac{k}{\mu} \operatorname{grad}\right)=\Phi c \frac{\partial p}{\partial t}
$$

i.e. by introducing pressure difference:

$\Delta \mathrm{p}=\mathrm{p}_{\mathrm{i}}-\mathrm{p}$

$$
\operatorname{div}\left(\frac{k}{\mu} \operatorname{grad} \Delta p\right)=\Phi c \frac{\partial \Delta p}{\partial t}
$$

The solutions of eq. 1-2 depending on different initial and boundary conditions are known. Boundary conditions are mostly the rate - defined by Darcy's law - and the zero pressure gradient at the boundary, so the solution is the exponential integral (Ei). If steady-state conditions are assumed, then the solution in the radial/cylindrical system will be logarithmic. 


\section{THEORY}

Equations 1 and 2 will be applied to a reservoir volume with arbitrary shape and size, and solved by means of the Laplace transform. The consequence of the continuity law is extra emphasized here, which means, that in nature processes take place always without breaks and jumps. Changes are proportional to the driving force and forces in reality are always finite, consequently the intensity of changes is always finite too. If continuity law is hurt, the system's response will be wrong. A typical example for this is the paradox of Achilles and the turtle.
Let's assume, that in a radial system, in the $r \leq r_{1}$ domain and on its boundary pressure change depends entirely on time, i.e. $\Delta p_{1}(t)=\Delta p_{1}\left(r \leq r_{1}, t\right)$. Let's utilize, that in the given $V$ reservoir volume the volumetric average pressure approaches the current equilibrium pressure of the system, $\Delta p_{2}(t)$. The rate of this approach is determined by the governing law of irreversible thermodynamics. In this case the Laplace transformed solution matching initial and boundary conditions is:

$$
\overline{\Delta p}(r, s)=\overline{\Delta p}_{1} \bar{F}_{1}(r, s)+\overline{\Delta p}_{2} \bar{F}_{2}(r, s)
$$

where

$$
\begin{aligned}
& \bar{F}_{1}(r, s)=\frac{R\left[I_{0 r} K_{1 R}+I_{1 R} K_{0 r}\right]-r_{1}\left[I_{0 r} K_{1 r_{1}}+I_{1 r_{1}} K_{0 r}\right]}{R\left[I_{0 r_{1}} K_{1 R}+I_{1 R} K_{0 r_{1}}\right]-r_{1}\left[I_{0 r_{1}} K_{r_{1}}+I_{r_{1}} K_{0 r_{1}}\right]} \\
& \overline{\mathrm{F}}_{2}(\mathrm{r}, \mathrm{s})=\frac{\sqrt{\frac{\mathrm{s}}{\mathrm{K}}\left(\mathrm{R}^{2}-\mathrm{r}_{1}^{2}\right)}}{2(1+\mathrm{s} \tau)} \frac{\mathrm{I}_{0 \mathrm{r}} \mathrm{K}_{0 \mathrm{r}_{1}}-\mathrm{I}_{0 \mathrm{r}_{1}} \mathrm{~K}_{0 \mathrm{r}}}{\mathrm{R}\left[\mathrm{I}_{0 \mathrm{r}_{1}} \mathrm{~K}_{1 \mathrm{R}}+\mathrm{I}_{1 \mathrm{R}} \mathrm{K}_{0 r_{1}}\right]-\mathrm{r}_{1}\left[\mathrm{I}_{0 \mathrm{r}_{1}} \mathrm{~K}_{1 \mathrm{r}_{1}}+\mathrm{I}_{1 \mathrm{r}_{1}} \mathrm{~K}_{0 \mathrm{r}_{1}}\right]}
\end{aligned}
$$

The $\tau$ quantity in eq. 5 is the so called eigentime of the system, which expresses a relationship between space size, space parameters and time as follows:

$$
\tau=\int_{V} \frac{\Phi \mu c}{k h} d V
$$

If petrophysical parameters are constant in the investigated domain, then the eigentime of the system is proportional to the volume of the system and is independent of its shape.

In reservoir engineering the eigentime has an approximate magnitude of $1 \mu \mathrm{s} /$, or $1 \mathrm{~s} / 1000 \mathrm{~m}^{3}$. In case of several billion $\mathrm{m}^{3}$ reservoir-aquifer systems the eigentime is in the year range.

The eigentime of the system has a dominant role in the system's response to influences/impacts. Every material system develops its response to impacts in the range of its eigentime.

Knowing the inverse of eq. 4 and 5 the pressure distribution in the $\mathrm{V}$ reservoir volume can be determined via Duhamel's theory, in form of a convolution integral:

$$
\Delta(r, t)=\Psi_{1}(t) * f_{1}(r, t)+\psi_{2}(t) * f_{2}(r, t)
$$

where the convolution integral is:

$$
\Delta p_{1}(t) * f_{1}(r, t)=\int_{0}^{t} \Delta p_{1}(x) f_{1}(r, t-x) d x
$$

The spatial and time domain distribution of the pressure difference can be determined by applying the expansion theorem [1]. By means of it the solution is obtained in form of infinite series. Beside the exactly inverted form it is worthwhile to investigate special forms of it, namely the asymptotic solutions.

\section{INVERSE TRANSFORMATION}

It can be proved, that functions $\bar{F}_{1}$ and $\bar{F}_{2}$ have only simple poles and the roots are purely imaginary. The poles of the two functions are identical, the only difference is that the pole

$\mathrm{s}=-1 / \tau$ is also root of $\bar{F}_{2}$, but it is not a root of $\bar{F}_{1}$. The solution of the starting PDEs (eqs.1-2) matching initial and boundary conditions consists of two parts:

- a transient part, which depends on the properties of the system only, and which is independent of timely impacts

- the quasi steady-state part, which also depends on the timely evolution of system impacts/influences.

In fluid recovery processes impacts/influences are mostly aperiodic in nature. In certain time intervals influences have constant intensity, which means, that they can be approximated with step (Heaviside) functions. Under special circumstances periodic impacts can also occur, for example in underground gas storage or pulse well testing, etc.

The most important among influences (bearing physical meaning) are the Dirac delta impulse, $[\delta(t)]$, the unit step (Heaviside) function [ $1(t)$ ] and the linear influences $[1(t) \times t]$. 
The response given by linear PDEs to Dirac delta are called weight functions, in our case $f_{1}(r, t)$ and $f_{2}(r, t)$. The response given to the Heaviside unit step function is called transfer function, whereas the response given to the timely linear function is the transmission function. These functions have the following relationships:

$$
\begin{aligned}
& g_{1}(r, t)=\int_{0}^{t} f_{1}(r, x) d x \\
& g_{2}(r, t)=\int_{0}^{t} f_{2}(r, x) d x \\
& h_{1}(r, t)=\int_{0}^{t} g_{1}(r, x) d x \\
& h_{2}(r, t)=\int_{0}^{t} g_{2}(r, x) d x
\end{aligned}
$$

\section{WEIGHT FUNCTIONS}

The system's response given to the Dirac delta impulse is called weight function. It determines, what is the contribution of the impact taking place at time $\xi$ in the response taking place some time later - at time $t-\xi$. From the inverted solution it can be seen, that functions $f_{l}$ and $f_{2}$ start at $t=0$ from nil. At small times $(t / \tau<<1) f_{1}$ and $f_{2}$ grows monotonically with time. Later they have an inflection and achieve their maximal value at around the eigentime, i.e. at $t / \tau \approx 1$. If time is increasing further $(t / \tau$ $\rightarrow \infty$ ) they tend to zero exponentially. Their integral from zero to infinity is unity, which is characteristic for weight functions. (This is due to $g(t \rightarrow \infty)=1$ ). From the nature of the weight functions it follows, that the system forms its response to impacts by weighting the elementary influences. The influence/impact acting at time $\mathrm{t}[\xi=\mathrm{t}]$ does not contribute to the system's response at all, because the value of the weight function at $t-\xi=0$ is zero.

In the dual (material and wave) nature world information propagates in form of waves and with finite velocity. On boundaries waves will be reflected. To form the response the system has to prepare itself. In the current system's response the elementary influences happened with eigentime earlier play the greatest role $[f(r, \tau) \rightarrow \max$.$] ,$ whereas very old happenings $[t / \tau \rightarrow \infty]$ have less and less contribution to the response. Steady-state is achieved if a constant intensity impact acts to the system sufficiently longer then the system's eigentime.

By definition the transfer function is equal to the system's response given to the unit step impact, i.e. to the time integral of the weight function. The transfer function determines the dynamic behavior of the system, because the system's response can be calculated with it for arbitrary influences. Transfer functions are the mathematical tools to describe turn-on and switch-off phenomena.

\section{SPATIAL DISTRIBUTION OF WEIGHT FUNCTIONS}

The spatial distribution of the pressure is determined by the Laplace transformed weight and transfer functions (eqs. 4,5). In the boundary domain of the impact $\left(r \rightarrow r_{1}\right)$ local behavior is described dominantly by the function $\mathrm{f}_{1}(\mathrm{r}, \mathrm{t})$, i.e. its Laplace transform $\bar{F}_{1}(r, s)$, because of $\bar{F}_{2}\left(r \rightarrow r_{1}, s\right) \rightarrow 0$. On the other hand the average pressure behavior of the whole system approaching to the equilibrium pressure is dominated by the $\bar{F}_{2}(r, s)$ weight function. (The volumetric integral of $\bar{F}_{1}$ is proportional to $r_{1}^{2} / R^{2}$, whereas the same integral of $\bar{F}_{2}$ is proportional with $1-r_{1}^{2} / R^{2}$.) If the system is influenced by a constant intensity long lasting impact (longer than the eigentime), then the system's response becomes steady-state, because

$$
\bar{F}_{1}(r, s \rightarrow 0)+\bar{F}_{2}(r, s \rightarrow 0) \rightarrow 1
$$

Material systems respond to impacts with some time delay. Response evolves earlier close to the impact's acting surface and then later in domains far away from it. Material systems behave initially as they were infinite. Due to the propagation and reflection of waves material systems build up their response on their eigentime scale. The fare we are from the acting surface of influences, the larger the system which has to build up a response, consequently its eigentime increases. Mathematically it means that the systems response is time and space dependent.

\section{CHARACTERISTIC TIME SCALES}

In reservoir engineering initial transient processes are also important. The exact solution describing these initial processes is generally in the form of slowly converging infinite series. In such cases deriving approximate solutions can reduce calculation time significantly.

For most of the problems those solutions are needed, which describe reservoir processes in the eigentime range, e.g. material balance calculations, multiple reservoir-aquifer interference calculations or well tests. Interaction or interference of reservoirs generates inflection points on pressure distribution curves. Propagation velocity of inflection points is inversely proportional to the squareroot of time: 


$$
v_{\mathrm{inf} l}=\frac{1}{\sqrt{1+\text { const } \times t}}
$$

The time scale which is much longer as the eigentime corresponds to steady-state conditions. If eqs. 4-5 are investigated at large times, i.e. $t \rightarrow \infty,(s \rightarrow 0)$, then invoking the asymptotic forms of Bessel functions we get:

$$
\begin{aligned}
& \bar{F}_{1}(r, s \rightarrow 0) \approx 1-\frac{\frac{R}{r_{1}}-1}{\sqrt{\frac{R}{r_{1}}} \ln \sqrt{\frac{R}{r_{1}}}} \ln \frac{r}{r_{1}} \ldots \ldots . . \\
& \bar{F}_{2}(r, s \rightarrow 0) \approx \frac{\frac{R}{r_{1}}-1}{\sqrt{\frac{R}{r_{1}}} \ln \sqrt{\frac{R}{r_{1}}}} \ln \frac{r}{r_{1}}
\end{aligned}
$$

It can be seen, that we obtain the well known logarithmic pressure distribution. If $\Delta \mathrm{p}_{1} \neq \Delta \mathrm{p}_{\infty}$, the pressure distribution becomes logarithmic, whereas if $\Delta p_{1}=\Delta p_{\infty}$, i.e. there are no influences at all, pressure distribution becomes homogeneous.

\section{WELL TEST MODELS}

Prior to welltests, in reservoirs production/injection activities are running. As a result of it the average equilibrium reservoir pressure - and that of the investigated reservoir domain - may, have changed. Pressure inhomogeneities in the adjacent reservoir domains tend to decline in form of fluid flows between domains. In any domain pressure approaches the equilibrium pressure of the domain. The rate of pressure change depends on the domain's eigentime and applied influences.

In well tests $\mathrm{R}$ means the distance of the observation well from the generator (pulsing, producing, etc.) well, whereas $r_{1}$ means the wellbore radius of the generator well. Physically seen well test evaluation models are the dynamic responses of the investigated domains to applied impact sequences, and from mathematical point of view they are the transfer functions of multiple, unit step function like impacts (sequences of constant rate injections/productions). In brief: well test models are the transfer functions of the system determining its response to Heaviside type unit step function influences. If influences/impacts are more complicated, then the system's response can be calculated by means of the convolution integral.
Well tests can be distinguished by the number of wells participating in the test. Onewell tests give information from the vicinity of the well, whereas multiwell tests provide information from reservoir properties between the wells.

In onewell tests the amplitude of the pressure change is larger as in the multiwell tests, because as the pressure wave propagates towards the observation well, friction causes its amplitude to decline.

For the different hydrodynamic tests analytical and numerical simulation models can be applied.

\section{ANALYTICAL SOLUTION}

For the evaluation of one- or multiwell hydrodynamic tests causing small pressure changes and lasting only short, analytical models based on the transient flow equation are adequate. These are for example pressure buildup, pressure drawdown, pulse and interference testing, reservoir limit tests, etc.

The basis of the solution is the time integral of the system's transfer function. In the mathematical solution the signal generated by the disturbing well crosses the reservoir volume extending to the observation wells, so the eigentime will be determined by the size and properties of this reservoir volume. As the amplitude of the generated signal declines as it propagates, at large distances (large volumes) the possibility to record the signal properly strongly reduces. In practice, in observation wells, which are a few $100 \mathrm{~m}$ far away, the signal (its shape, its amplitude and evolution) still can be recorded. From this it follows, that the eigentime of this reservoir volume including this few $100 \mathrm{~m}$ is in the range of a few hours (or less), which is still a fraction of the whole system's eigentime. It means, that the duration of signal generation in well tests is in the range of the eigentime of the sub-system (investigated reservoir domain), or even it is larger. The mathematical consequence is, that in the model the exact solution of the weight and transfer functions is necessary, and it is not enough to know the asymptotic solution.

In well tests the generated signal appears with some time lag and amplitude modulated in the observation well, depending on the eigentime. The inverse i.e. time integral (transfer function) of weight functions (eqs. 4,5) can be determined by the expansion theorem [1]. The transcendental equation providing the common roots is:

$$
\left.\lambda J\left(\lambda \alpha_{n}\right) Y_{0}\left(\alpha_{n}\right)-J_{0}\left(\alpha_{n}\right) Y_{1}\left(\lambda \alpha_{n}\right)\right]-\left[J_{1}\left(\alpha_{n}\right) Z_{0}\left(\alpha_{n}\right)-J_{0}\left(\alpha_{n}\right) Y_{1}\left(\alpha_{n}\right)\right]=0(13)
$$

where $\alpha_{n}$ are the roots of the above equation, $n=1,2,3$..

The weight function of the system in form of infinite series is: 
$f_{1}(r, t)=\sum_{n=1}^{\infty} \frac{2 \alpha_{n} \pi\left(\lambda^{2}-1\right)}{\lambda \tau} Q_{1 n} e^{-\alpha_{n}^{2} \pi\left(\lambda^{2}-1\right) t / \tau}$

$f_{2}(r, t)=\frac{1}{2 \tau} \sqrt{\frac{\lambda^{2}-1}{\pi}} Q_{21} e^{-t / \tau}+\sum_{n=1}^{\infty} \frac{\alpha_{n}^{2} \pi\left(\lambda^{2}-1\right)}{\lambda \tau\left[\alpha_{n}^{2} \pi\left(\lambda^{2}-1\right)-1\right]} Q_{2 n} e^{-\alpha_{n}^{2} \pi\left(\lambda^{2}-1\right) t / \tau}$

where

$Q_{1 n}=\frac{\lambda\left[J_{0}\left(\alpha_{n} r_{D}\right) Y_{1}\left(\lambda \alpha_{n}\right)-J_{1}\left(\lambda \alpha_{n}\right) Y_{0}\left(r_{D} \alpha_{n}\right)\right]-\left[J_{0}\left(r_{D} \alpha_{n}\right) Y_{1}\left(\alpha_{n}\right)-J_{1}\left(\alpha_{n}\right) Y_{0}\left(r_{D} \alpha_{n}\right)\right]}{\lambda\left[J_{0}\left(\lambda \alpha_{n}\right) Y_{0}\left(\alpha_{n}\right)-J_{0}\left(\alpha_{n}\right) Y_{0}\left(\lambda \alpha_{n}\right)\right]-\left[J_{1}\left(\lambda \alpha_{n}\right) Y_{1}\left(\alpha_{n}\right)-J_{1}\left(\alpha_{n}\right) Y_{1}\left(\lambda \alpha_{n}\right)\right]}$

$Q_{21}=\frac{J_{0}\left(\beta r_{D}\right) Y_{0}(\beta)-J_{0}(\beta) Y_{0}\left(\beta r_{D}\right)}{\lambda\left[J_{1}(\lambda \beta) Y_{0}(\beta)-J_{0}(\beta) Y_{1}(\lambda \beta)\right]-\left[J_{1}(\beta) Y_{0}(\beta)-J_{0}(\beta) Y_{1}(\beta)\right]}$

$Q_{2 n}=\frac{J_{0}\left(\alpha_{n} r_{D}\right) Y_{0}\left(\alpha_{n}\right)-J_{0}\left(\alpha_{n}\right) Y_{0}\left(\alpha_{n} r_{D}\right)}{\lambda\left[J_{0}\left(\lambda \alpha_{n}\right) Y_{0}\left(\alpha_{n}\right)-J_{0}\left(\alpha_{n}\right) Y_{0}\left(\lambda \alpha_{n}\right)\right]-\left[J_{1}\left(\lambda \alpha_{n}\right) Y_{1}\left(\alpha_{n}\right)-J_{1}\left(\alpha_{n}\right) Y_{1}\left(\lambda \alpha_{n}\right)\right]}$

The transfer functions, which determine the system's

response to Heaviside type unit step influences are as

follows:

$$
\begin{aligned}
& g_{1}(r, t)=1-\sum_{n=1}^{\infty} \frac{2}{\lambda \alpha_{n}} Q_{1 n} e^{-\alpha_{n}^{2} \pi\left(\lambda^{2}-1\right) t / \tau} \\
& g_{2}(r, t)=-\frac{1}{2} \sqrt{\frac{\lambda^{2}-1}{\pi}} Q_{21} e^{-t / \tau}-\sum_{n=1}^{\infty} \frac{\lambda^{2}-1}{\lambda\left[\alpha_{n}^{2} \pi\left(\lambda^{2}-1\right)-1\right]} Q_{2 n} e^{-\alpha_{n}^{2} \pi\left(\lambda^{2}-1\right) t / \tau}
\end{aligned}
$$

For $t / \tau<<1$ these series converge slowly, so in this case they can be approximated with their asymptotic form:

$$
\begin{aligned}
& f_{1}(r, t / \tau \ll 1) \approx \frac{1}{\sqrt{r_{D} \pi}}\left[\frac{\xi^{2}}{t} e^{-\xi^{2}}-\frac{1}{\sqrt{\lambda}} \frac{\eta}{t} e^{-\eta^{2}}\right] \\
& f_{2}(r, t / \tau \ll 1) \approx \frac{1}{\sqrt{r_{D}}} \frac{1}{2 \pi} \sqrt{\frac{\lambda^{2}-1}{\lambda \pi t}} e^{-\eta^{2}} .
\end{aligned}
$$

The exact solutions of weight and transfer functions of the system depend only on the eigentime and radius ratio, so type curves can be generated for the whole time scale. Knowing the weight and transfer functions the system's response to any arbitrary impact or influence can be determined.

There are two characteristic cases in practice. In the first one we assume, that the induced pressure change is stepwise like. In this case the system's response is the transfer function given by eqs. 19-20. They have to be applied considering the shifting theorem of Laplace transformation due to the periodic nature of the impact. In the second case - which is more general in practice - signal generation is accomplished by periodic or aperiodic, but impulse like change of production/injection rates in the disturbing well. In this instant the generated signal will not be impulse type, because depending on the properties of the well vicinity its shape and evolution will be deformed. The system's response can be determined also in this case by either applying the expansion theorem or, by the convolution integral.

Let's suppose, that in the signal generator well the signal is generated by periodic constant rate production or injection. The impact series can now be approximated by the Heaviside type unit step functions as follows:

$$
q(t)=\sum_{i=0}^{n}\left[1\left(t-T_{i}\right)-1\left(t-T_{i+1}\right)\right] g_{i}
$$

where

$q_{i}=$ constant rate in the interval $T_{i} \leq t \leq T_{i+1}$

$T_{i}=$ timepoints where the rate changes

If in eq.23. every second rate is the same and their time periods are also the same, then we have a periodic, otherwise an aperiodic signal generation. If $\mathrm{q}_{i}=0, i \neq 0$, then we have a pressure buildup or pressure falloff test. 
The Laplace transform of eq. 23 is:

$$
\bar{q}(s)=\sum_{i=0}^{n} \frac{q_{i}}{s}\left[e^{-s T_{i}}-e^{-s T_{i+1}}\right]
$$

Let's utilize, that at the location of signal generation ( $r=$ $r_{1}$ ) pressure gradient and rate are related as by Darcy's law:

$$
\bar{q}=-\frac{k}{\mu} 2 r_{1} \pi h\left(\frac{d \bar{p}}{d r}\right)_{r_{1}}
$$

Considering eqs. 24 and 25 the Laplace transformed pressure change is:

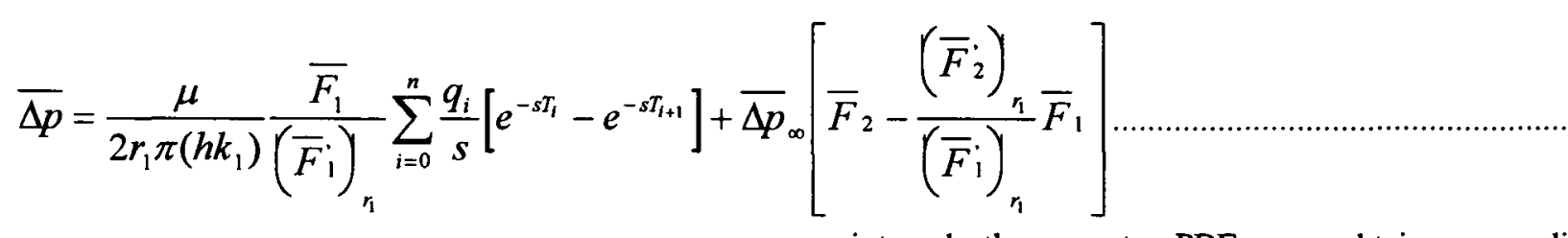

Similarly to the transfer function, the inverse of eq. 26. can be determined by the expansion theorem, resulting in an infinite series.

During well tests the system's equilibrium pressure is practically constant. If several wells participate in the test, then we obtain information from the reservoir properties in the vicinity of the signal generator well, further from the reservoir domain between generator and observation wells. The signal generated with production/injection propagates in the reservoir in wave form, and during traveling its amplitude declines according to those reservoir properties which characterize the passed reservoir sections. This useful signal can be distorted by the pressure trend of the observation wells, which tend towards their current equilibrium pressure. This so called "noise" is physically the pressure decline of wells towards their current equilibrium pressure, which was caused by earlier impacts. Whether a measurement can be evaluated or not, depends on the ratio of useful signal to "noise".

\section{SIMULATION SOLUTION}

Simulation solutions can be suggested in case of multiple, vertical, deviated or horizontal wells with long duration signal generation causing relatively larger pressure amplitudes, where pressure recording is possibly not at the perforation depth, but higher.

If simulation grids are chosen sufficiently small, and the eigentime of them is small compared to production time, then the response of the sub-system (gridblock) becomes quasi steady-state. By subdivision the resulting small domains can be characterized by their average pressure. Their response to impacts is determined by their eigentimes. Whether we want to investigate the evolution of pressure or fluid composition in time, by applying the integral theorem to PDE, we obtain an ordinary differential equation (ODE), whose general form is:

$$
\frac{d y}{d t}=\frac{y_{\infty}-y}{\tau}
$$

where $y(t)$ is the target function of the system for the given timepoint. Depending on what we want to investigate, the target function is either the equilibrium pressure of the system, or the equilibrium fluid composition.

Eq. 27 can be readily solved either by the integrating factor method or by Laplace transform. The system tries his state variable to approach the target function. During timesteps the target function can be approximated linearly or with a higher order polynomial. For these kind of target functions eq. 27. can be readily solved, so the timely evolution of the state variable can be calculated analytically and explicitly.

If the volume and consequently the eigentime of the investigated subdomain is small compared to the time interval of the changing target function, then the quasi steady-state solution follows the target function with a time lag equal to the eigentime:

$$
y(t) \approx y_{\infty}(t-\tau) \quad t / \tau>1
$$

It is true of course, that if a large volume is subdivided to small volumes (grids in simulation), then the eigentime of the large system is the sum of the eigentimes of the small subdomains. The time lag of the whole system is proportional to the sum of the time delays of the sub systems (i.e. domains), because the volume integral is additive.

In conventional simulation methods by discretizing space and time - which is allowed mathematically - the continuity and impulse change laws are hurt, so the calculated system's response may be distorted. State variables have in this case breaks and jumps, which is physically impossible. We have proved, that material 
systems respond to even Dirac type (peak) pulses with a continuous answer.

As subsystems (gridblocks) are characterized by their eigentimes - which can be calculated by a simple integral - determining their dynamic behavior, the blocks can have of any irregular shape, orthogonality is not a requirement, so girding has a very high degree of freedom.

In the mathematical and physical model we have assumed and utilized apriori, that with impacts/influences we generate thermodynamic forces, to which systems have to respond continuously and without any jumps or breaks. The response of the gridblocks with irregular shape and arbitrary size can be calculated fully explicitly with arbitrary timesteps depending only on the influence. In the timestep itself the solution can be calculated analytically by solving an $\mathrm{ODE}$.

In case of complex well patterns and completions girding can be arbitrarily dense in the vicinity of wells. Grids can have e.g. flexible triangular shape, similar to finite element solutions. Knowing influences (production/injection) grid responses can be calculated fully explicitly. The spatial distribution of petrophysical parameters can be recalculated from the eigentimes of the blocks. These are actually the history matching parameters. Due to the flexible girding and to the fully explicit calculation scheme very high calculation speeds can be achieved.

The well test simulator is actually the same as a full field simulator, the only difference is being in the nature of the grid and the target function. In application to well tests the shape of the generated signal does not complicate the use of the simulator, because the model is "life-path" type, where influences are impulse type, but the system's response can always be continuous only.

\section{SUMMARY}

A new, general mathematical model has been developed and applied to well tests. Both analytical and numerical applicability have been presented. The concept of eigentime has been introduced.

Some characteristic features of the model are as follows:

- in well tests the signal generation can be of any shape and duration

- the reservoir's i.e. the model's response to impacts and influences is always continuous

- the model simultaneously estimates reservoir properties near the wellbore and up to the observation well.
- the model generates meaningful responses for the whole time scale, i.e. at times much less, nearly equal and much higher as the eigentime

- reservoir "noise" can be soundly described by the model, "noise" is the pressure change of wells, as they approach their current equilibrium pressure

- type curves can be readily generated by the model

- in applying the model to numerical simulations , arbitrary shaped grids can be used with no need to orthogonality

- the solution scheme used is fully explicit with minor constraint to the timestep size.

\section{NOMENCLATURE}

$$
\begin{aligned}
& \text { c = compressibility } \\
& f_{1,2}(t)=\text { weight functions } \\
& \mathrm{g}_{\mathrm{I}, 2}(\mathrm{t})=\text { transfer function } \\
& \mathrm{h}_{1,2}(\mathrm{t})=\text { transmission function } \\
& \bar{F}(s) \text { = Laplace transformed function } \\
& \text { h = formation thickness } \\
& \text { i = imaginary unit } \\
& \mathrm{I}_{0} \quad=\text { modified, first kind, zero order } \\
& \text { Bessel function } \\
& \mathrm{I}_{1} \quad=\text { modified, first kind, first order } \\
& \text { Bessel function } \\
& \mathrm{J}_{0} \quad=\text { first kind, zero order Bessel } \\
& \text { function } \\
& \mathrm{J}_{1} \quad=\text { first kind, first order Bessel } \\
& \text { function } \\
& \mathrm{K}_{0} \quad=\text { modified, second kind, zero order } \\
& \text { Bessel function } \\
& \mathrm{K}_{1} \quad=\text { modified, second kind, first order } \\
& \text { Bessel function } \\
& \mathrm{k}=\text { permeability } \\
& \mathrm{K}=\mathrm{k} /(\Phi \mu \mathrm{c}) \\
& \mathrm{p} \quad=\text { pressure } \\
& \mathrm{q} \quad=\text { rate } \\
& r_{1}, R \quad=\text { internal and external radius } \\
& r_{D}=\text { dimensionless radius }\left(r / r_{1}\right) \\
& \text { s = Laplace parameter } \\
& \mathrm{V} \quad=\text { velocity } \\
& \mathrm{V} \quad=\text { volume } \\
& \mathrm{y} \quad=\text { target function } \\
& \mathrm{Y}_{0} \quad=\text { second kind, zero order Bessel } \\
& \text { function } \\
& \mathrm{Y}_{1} \quad=\text { second kind, first order Bessel } \\
& \text { function } \\
& =\text { time } \\
& =\text { eigentime } \\
& \begin{array}{ll}
\mu & =\text { viscosity } \\
\Phi & =\text { porosity }
\end{array}
\end{aligned}
$$$$
\alpha_{n}=i \sqrt{\frac{s_{n}}{K}} r_{1} \text {, roots of transcendent equation }
$$

$\lambda=$ relative distance, $R / r_{1}$

$\delta(t) \quad=$ Dirac delta function 


$$
\begin{aligned}
& \beta=\frac{1}{\sqrt{\pi\left(\lambda^{2}-1\right)}} \\
& \xi^{2}=\frac{\left(r_{D}-1\right)^{2} \tau}{4 \pi\left(\lambda^{2}-1\right) t} \\
& \eta^{2}=\frac{\left(\lambda-r_{D}\right)^{2} \tau}{4 \pi\left(\lambda^{2}-1\right) t}
\end{aligned}
$$

\section{Subscripts}

$$
\begin{array}{ll}
\infty & =\text { equilibrium } \\
\text { infl } & =\text { inflection }
\end{array}
$$

\section{Superscripts}

- Laplace transformed

\section{ACKNOWLEDGMENT}

We appreciate the support of MOL Plc. to publish this paper.

\section{REFERENCES}

1. Fodor, Gy:: Technical Application of the Laplace Transform (in Hungarian) Techn.Ed., Budapest, 1966. 